\title{
MITRAL REGURGITATION CAUSED BY RUPTURED CHORDAE TENDINEAE IN KAWASAKI DISEASE
}

\author{
Akira Mishima, MD, Miki Asano, MD, Takayuki Saito, MD, Shigeki Yamamoto, MD, Tomohiko Ukai, MD, \\ Hirohisa Yoshitomi, MD, Kozo Mastumoto, MD, and Tadao Manabe, MD, Nagoya, Japan
}

Kawasaki disease, a generalized vasculitis of unknown etiology, is becoming one of the leading causes of acquired heart disease in children. In the acute phase of illness, cardiac findings may reveal the involvement of the pericardium, myocardium, endocardium, and coronary arteries. Complications involving the coronary arteries are the most common causes of both early and late morbidity and mortality. ${ }^{1}$ Valvular heart disease, such as primary mitral regurgitation, rarely occurs as a complication and may result in severe congestive heart failure. We report a case of mitral regurgitation caused by ruptured chordae tendineae in a patient with Kawasaki disease who underwent successful valve repair. Rupture of the chordae tendineae has not been previously reported as a cause of mitral regurgitation in Kawasaki disease.

The patient's Kawasaki disease was diagnosed at 3 months of age, when he had 5 days of fever, truncal rash, erythema of the palms and lips, bilateral conjunctival injection, and cervical lymphadenopathy. His serum C-reactive protein concentration was $9.1 \mathrm{mg} / \mathrm{dl}$. He was treated 6 days after the onset of illness with high-dose aspirin and intravenous gamma globulin at a dosage of $400 \mathrm{mg} / \mathrm{kg}$ per day for 3 days. The patient's clinical features improved rapidly, and his serum $\mathrm{C}$-reactive protein concentration decreased to $0.7 \mathrm{mg} / \mathrm{dl}$. His condition remained stable until day 24 of his illness, when a II/VI apical systolic murmur suddenly developed and his serum C-reactive protein concentration increased to $6.8 \mathrm{mg} / \mathrm{dl}$. The patient had no fever at that time. Two-dimensional echocardiography and Doppler examination demonstrated mild mitral regurgitation without appreciable abnormalities of the coronary arteries. During a 3-day period, the patient came to have congestive heart failure as a result of mitral regurgitation. Intravenous gamma globulin was again administered to treat a recurrence of acute Kawasaki disease. Evaluation indicated rapid resolution of systemic inflammation, but severe heart failure resulting from significant mitral regurgitation persisted.

Three months after the onset of illness, the patient was referred to our hospital for further evaluation. Cardiac catheterization confirmed the presence of grade $4 / 4$ mitral regurgitation with associated pulmonary hypertension and a markedly dilatated left ventricle without evidence of hypokinesia or akinesia. Coronary angiography revealed

From the First Department of Surgery, Nagoya City University Medical School, Nagoya, Japan.

Received for publication August 22, 1995; accepted for publication August 29, 1995.

J ThoraC Cardiovasc Surg 1996;111:895-6

Copyright (C) 1996 by Mosby-Year Book, Inc.

$0022-5223 / 96 \$ 5.00+0 \quad \mathbf{1 2 / 5 4} / \mathbf{6 9 2 6 0}$ normal coronary arteries. The pulmonary artery pressure was $78 / 35 \mathrm{~mm} \mathrm{Hg}$ and the pulmonary artery resistence index was calculated to be $7.8 \mathrm{U} \cdot \mathrm{m}^{2}$.

At 12 months of age, the patient underwent successful mitral valve repair for symptoms of persistent heart failure and poor weight gain. The middle portion of the posterior leaflet of the mitral valve was slightly thickened and prolapsed. This prolapse was caused by rupture of the chordae tendineae attached to the free edge of the valve. The papillary muscles and the anterior leaflet of the mitral valve appeared normal. The posterior leaflet underwent quadrangular resection at the prolapsed site, and the gap created by the resected posterior leaflet was corrected by annular plication. A total circular annuloplasty was performed to repair the enlarged mitral orifice, resulting in an orifice diameter of $14 \mathrm{~mm}$. Intraoperative Doppler echocardiography indicated trace mitral regurgitation. Histologic examination of the excised mitral valve leaflet revealed the abscence of valvulitis. The patient had an uneventful postoperstive recovery, and cardiac catheterization was performed 3 weeks after operation. Left ventriculography demonstrated the complete absence of mitral regurgitation. Pulmonary artery pressure and pulmonary artery resistance index were reduced to $40 / 15 \mathrm{~mm}$ $\mathrm{Hg}$ and $3.5 \mathrm{U} \cdot \mathrm{m}^{2}$, respectively.

It is well known that mitral regurgitation occurs in the acute phase of Kawasaki disease as a result of acute valvulitis, papillary muscle dysfunction, or myocardial infarction. ${ }^{2-5}$ Mild, inaudible mitral regurgitation is frequently detected by Doppler echocardiography during the acute phase of illness but rapidly improves. This valvular disease may be a result of papillary muscle dysfunction caused by a pancarditis. ${ }^{2}$ Although the mechanism of myocarditis in Kawasaki disease is unknown, autoimmune mechanisms may be responsible for the changes seen in myocardial structures. Because of the functional reversibility of the myocarditis seen in Kawasaki disease, the target of autoimmune attack is suggested to be a relatively renewable structure, such as an intramyocardial microvessel. ${ }^{1}$ In contrast, mitral regurgitation with an audible murmur has been described in about $1 \%$ of patients with Kawasaki disease. Chronic mitral regurgitation may be caused by involvement of the valve and its associated papillary muscles as a result of valvulitis or coronary artery stenosis. ${ }^{3}$ Surgical repair for persistent mitral regurgitation resulting from Kawasaki disease has been described as difficult because of the high incidence of severe associated ischemic heart disease. When required, the valvular disease has occasionally been treated through surgical intervention such as mitral annuloplasty or valve replacement. 4,5

In our patient, a favorable clinlcal response to the initial therapy was achieved in the acute phase of Kawasaki 
disease and the coronary arteries were not affected. On day 24 of the illness, however, mitral regurgitation with an audible murmur occured suddenly, resulting in the rapid development of severe heart failure. Doppler echocardiography demonstrated prolapse of the posterior leaflet of the mitral valve, which before operation was attributed to rupture of the papillary muscle in the setting of recurrent myocarditis. Rupture of the chordae tendineae was confirmed at the time of operation; however, there was no evidence of valvulitis. The valve was successfully repaired by mitral valvuloplasty with total circular annuloplasty.

Results of this case suggest that initial mild mitral regurgitation may be attributable to papillary muscle dysfunction caused by recurrent myocarditis. Severe mitral regurgitation, however, may be caused by chordal rupture that occurs because of functional impairment of the valve apparatus. This is the first report of mitral regurgitation resulting from ruptured chordae tendineae in a patient with Kawasaki disease. Evaluation of similar patients will be necessary to investigate in greater detail mechanisms responsible for chordal rupture.

\section{REFERENCES}

1. Takahashi M. Myocarditis in Kawasaki syndrome: a minor villian? Circulation 1989;79:1398-400.

2. Suzuki A, Kamiya T, Tsuchiya K, et al. Tricuspid and mitral regurgitation detected by color flow Doppler in the acute phase of Kawasaki disease. Am J Cardiol 1988;61:386-90.

3. Akagi T, Kato H, Inoue O, Sato N, Imamura K. Valvular heart disease in Kawasaki syndrome: incidence and natural history. Am Heart J 1990;120:366-72.

4. Konishi $\mathrm{Y}$, Tastuta N, Miki S, et al. Mitral insufficiency secondary to mucocutaneous lymph node syndrome: a case report of successful surgical treatment. Jpn Circ J 1978;42: 901-9.

5. Gidding SS, Shulman ST, Ilbawi M, Crussi F, Duffy CE. Mucocutaneous lymph node syndrome (Kawasaki disease): delayed aortic and mitral insufficiency secondary to active valvulitis. J Am Coll Cardiol 1986;7:894-7.

\title{
LATE FAILURE OF INTERNAL THORACIC ARTERY GRAFTS CAUSED BY SEQUELAE OF MEDIASTINITIS OR ITS TREATMENT
}

\author{
Luc Noyez, MD, Stefan H. Skotnicki, MD, and Leon K. Lacquet, MD, Nijmegen, The Netherlands
}

Mediastinitis is a severe complication after cardiac operations, leading to higher morbidity and mortality rates and to greatly increased hospital costs. The risk for erosion and hemorrhage of bypass grafts and the heart is known, but it is generally accepted that mediastinitis does not affect long-term graft patency. ${ }^{1}$ The following case report, however, is suggestive of occlusion of internal thoracic artery (ITA) grafts as a late result of mediastinitis or its treatment.

In 1987, a 56-year-old man underwent elective primary myocardial revascularization with both ITAs: left ITA to the diagonal branch (side to side) and left anterior descending coronary artery (end to side), the right ITA, retroaortic to the circumflex coronary artery, and a single vein graft to the right coronary artery. The first postoperative day, the patient was extubated and discharged from the intensive care unit. Six days after operation, the

From the Department of Thoracic and Cardiac Surgery, University Hospital Nijmegen St. Radboud, Nijmegen, The Netherlands.

Received for publication Sept. 19, 1995; accepted for publication Oct. 9, 1995.

J THOrac Cardiovasc Surg 1996;111:896-7

Copyright $(1) 1996$ by Mosby-Year Book, Inc.

$0022-5223 / 96 \$ 5.00+0 \quad \mathbf{1 2 / 5 4 / 6 9 7 4 8}$ patient had an unstable sternum. There were no signs of infection (fever, leukocytosis, wound drainage), and a refixation was performed. Four days later, the patients was septicemic, with a temperature of more than $40^{\circ} \mathrm{C}$ and leukocytosis. The sternal wound was producing purulent fluid and there was sternal dehiscence. Surgical exploration confirmed the diagnosis of mediastinitis with purulent fluid. Staphylococcus epidermidis was found to be responsible for the infection. Debridement of the mediastinum and sternum, insertion of a suction-irrigation tube, and reclosure of the sternotomy were performed. General and local antibiotic therapy were started. Because the patient remained septicemic, reexploration was performed 2 days later. After radical debridement of the mediastinum and a sternectomy, the greater omentum was transposed and wrapped around the heart, and the wound was treated open. Antibiotic therapy was continued. There was a good response to this therapy. A clean wound developed, and secondary closure of the wound was performed 22 days after the last intervention. The patient was discharged from the hospital after 38 days. The wound was healed without evidence of infection. The patient was seen regularly in the outpatient clinic. There were no wound problems. In 1989, the patient reported atypical retrosternal pain. An angiogram showed three patent grafts.

In January 1995, the patient reported angina (Canadian Heart Association class 2 to 3) and received medical 